\title{
A homological definition of the HOMFLY polynomial
}

\author{
STEPhen Bigelow
}

\begin{abstract}
We give a new definition of the knot invariant associated to the Lie algebra $\mathfrak{s u}_{N+1}$. Knowing these for all $N$ is equivalent to knowing the HOMFLY polynomial. Our definition requires that the knot or link be presented as the plat closure of a braid. The invariant is then a homological intersection pairing between two immersed manifolds in a configuration space of points in a disk. This generalizes previous work on the Jones polynomial, which is the case $N=1$.
\end{abstract}

57M25; 57M27, 20F36

\section{Introduction}

The Jones polynomial [7] was the first of the new generation of knot invariants, now called "quantum invariants". Soon after its discovery, several people independently discovered a two variable version, now usually referred to as the HOMFLY polynomial [5]. In this paper, we will be working with the invariant of type $A_{N}$, which can be obtained by specializing the HOMFLY polynomial $X_{L}(q, \lambda)$ to the value $\lambda=q^{N}$. One motivation for studying this specialization is that it is related to the representation theory of $\mathfrak{s u}_{N+1}$.

Fix an integer $N>1$, and let $P$ be the invariant of type $A_{N}$. This is an invariant of oriented knots and links that takes values in the $\mathbf{Z}\left[q^{ \pm 1 / 2}\right]$. It satisfies the following skein relation:

$$
q^{(N+1) / 2} P(\searrow)-q^{-(N+1) / 2} P(\nearrow)=\left(q^{1 / 2}-q^{-1 / 2}\right) P(\ \circlearrowright)
$$

Here, the three diagrams represent three links that are the same except inside a small ball, where they are as shown. We can define $P$ to be the unique invariant that satisfies the above skein relation and takes the value one for the unknot.

In [1], I presented a definition of the Jones polynomial as a homological intersection pairing between a certain pair of manifolds in a configuration space. The aim of this paper is to give a similar definition of the invariant of type $A_{N}$. The Jones polynomial is the special case $N=1$. The HOMFLY polynomial can be reconstructed from the values of all invariants of type $A_{N}$, which (perhaps) excuses the title of this paper. 
Suppose $\beta$ is a braid with $2 n$ strands and with orientations on the strands. Suppose the orientations are such that, reading from left to right along the bottom of $\beta$, the orientations are down, up, down, up, and so on, and reading from left to right along the top of $\beta$, the orientations are also down, up, down, up, and so on. Let $\widetilde{\beta}$ be the plat closure of $\beta$, obtained by joining adjacent pairs of nodes at the top and at the bottom of $\beta$. The orientations on strands of $\beta$ give consistent orientations to the components of $\widetilde{\beta}$. Every oriented knot or link can be obtained in this way.

The first goal of this paper is to define an invariant $Q(\beta)$. In Section 2, we define a configuration space $C$. This is similar to the space $C$ in [1] except that we assign colors to the puncture points and the points that make up a configuration. The colors determine which pairs of points are allowed to coincide, and how to compute the monodromy of a loop in the configuration space. In Section 3, we define immersed manifolds $T$ and $S$ in $C$. In Section 4, we define $Q(\beta)$ as an intersection pairing between $S$ and the image $\beta(T)$ of $T$.

The second goal of this paper is to prove that $Q(\beta)=P(\tilde{\beta})$. In Sections 5, 8, and 9, we prove that $Q(\beta)$ is invariant under certain moves. By a result of Birman [3], this implies that $Q(\beta)$ is an invariant of the oriented knot or link $\widetilde{\beta}$. The more difficult moves require some special tools, which we develop in Section 6 and Section 7. In Section 10, we prove that $Q(\beta)$ satisfies the above skein relation. In Section 11, we bring these results together to show that $Q(\beta)=P(\tilde{\beta})$.

Lawrence gave similar homological definitions of the Jones polynomial and the invariant of type $A_{N}$ in [8] and [9]. The definition here appears different, and includes a more precise description of the relevant manifolds in the configuration space. Under close examination, the two approaches might turn out to be the same.

One possible future application of this paper is to generalize the ideas in [10]. There, Manolescu gives evidence of a connection between the definition of the Jones polynomial in [1] and the invariant defined by Seidel and Smith in [11]. Both definitions involve intersections between manifolds in configuration spaces. However Seidel and Smith obtain the additional structure of a graded abelian group, which they conjecture to be a collapsed version of Khovanov's homology theory. It would be interesting to know whether the intersection pairing in [1] and in this paper could be refined to give a graded abelian group.

Acknowledgements This research was partly supported by NSF grant DMS-0307235 and Sloan Fellowship BR-4124. I am grateful to Ciprian Manolescu and Dylan Thurston for their interest and useful conversations. 


\section{Preliminaries}

In this section, we fix some notation and terminology. Throughout this paper, $N$ and $n$ will be fixed positive integers, and we let $k=2 n$, and $m=N n$. We also fix a unit complex number $q$ that is not a root of unity, and a choice of square root $q^{1 / 2}$. (We work over $\mathbf{C}$, but most of our construction works over a more general ring, as we briefly discuss at the end of the paper.)

\subsection{The configuration space}

Let $D$ be the unit disk in the complex plane, and let

$$
\mathbf{p}=\left(p_{1}, \ldots, p_{k}\right)
$$

be a sequence of $k$ points ordered from left to right on the real line in $D$. Let $\mathbf{c}$ be the $k$-tuple

$$
\mathbf{c}=\left(c_{1}, \ldots, c_{k}\right)=(0, N+1,0, N+1, \ldots, 0, N+1) .
$$

We use the notation $D_{\mathbf{c}}$ to denote the triple $(D, \mathbf{p}, \mathbf{c})$. We call this a disk with puncture points $p_{1}, \ldots, p_{k}$, having colors $c_{1}, \ldots, c_{k}$ respectively.

Let $\mathbf{c}^{\prime}$ be the $m$-tuple

$$
\mathbf{c}^{\prime}=\left(c_{1}^{\prime}, \ldots, c_{m}^{\prime}\right)=(1,2, \ldots, N, 1,2, \ldots, N, \ldots, 1,2, \ldots, N) .
$$

Let $\widetilde{C}_{\mathbf{c}^{\prime}}\left(D_{\mathbf{c}}\right)$ be the set of all $m$-tuples $\left(x_{1}, x_{2}, \ldots, x_{m}\right)$ of points in $D$ such that

- if $1 \leq i<j \leq m$ and $\left|c_{i}^{\prime}-c_{j}^{\prime}\right| \leq 1$ then $x_{i} \neq x_{j}$, and

- if $1 \leq i \leq m, 1 \leq j \leq k$, and $\left|c_{i}^{\prime}-c_{j}\right|=1$ then $x_{i} \neq p_{j}$.

Now let $W$ be the group of permutations $w$ of $\{1, \ldots, m\}$ such that $c_{i}^{\prime}=c_{w(i)}^{\prime}$ for all $i=1, \ldots, m$. Let $C_{\mathbf{c}^{\prime}}\left(D_{\mathbf{c}}\right)$ be the quotient of $\widetilde{C}_{\mathbf{c}^{\prime}}\left(D_{\mathbf{c}}\right)$ by the induced action of $W$.

Thus a point in $C_{\mathbf{c}^{\prime}}\left(D_{\mathbf{c}}\right)$ is a configuration of $m$ points in $D$, which we call mobile points. Each mobile point is assigned a color. Two mobile points of the same color are indistinguishable. A mobile point may coincide with a puncture point or another mobile point if and only if their colors differ by at least two.

We will occasionally use configuration spaces similar to $C_{\mathbf{c}^{\prime}}\left(D_{\mathbf{c}}\right)$ but with different sequences of colors $\mathbf{c}^{\prime}$ and $\mathbf{c}$. From now on, let $C$ denote $C_{\mathbf{c}^{\prime}}\left(D_{\mathbf{c}}\right)$. 


\subsection{Braids}

The braid group $B_{k}$ has many equivalent definitions, including: the mapping class group of a $k$-times punctured disk, the fundamental group of a certain configuration space, and the group of geometric braids with $k$ strands. We will move freely between these definitions. We will also allow some extra structure, such as colors or orientations on the strands of a geometric braid. Elements of the mapping class group act on the left, paths in a configuration space compose from left to right, and geometric braids read from top to bottom.

A braid in $B_{k}$ induces a permutation of the puncture points in $D_{\mathbf{c}}$. Let the mixed braid group $B_{\mathbf{c}}$ be the subgroup of $B_{k}$ consisting of braids that preserve the colors of the puncture points. Throughout this paper, $\beta$ will be a fixed element of $B_{\mathbf{c}}$.

Think of $\beta$ as a geometric braid and orient its strands in such a way that the upward strands are colored $N+1$ and the downward strands are colored 0 . The condition $\beta \in B_{\mathbf{c}}$ is equivalent to the condition on the orientations described in Section 1 . We can take the plat closure of $\beta$ to obtain an oriented knot or link $\widetilde{\beta}$. Let $P(\widetilde{\beta})$ be the invariant of $\widetilde{\beta}$ of type $A_{N}$.

We will represent elements of $\pi_{1}(C)$ using braids as follows. Let $\mathbf{c c}^{\prime}$ denote the concatenation

$$
\mathbf{c c}^{\prime}=\left(c_{1}, \ldots, c_{k}, c_{1}^{\prime}, \ldots, c_{m}^{\prime}\right) .
$$

Let $G$ be group of those mixed braids in $B_{\mathbf{c c}^{\prime}}$ whose first $k$ strands are straight. Then $\pi_{1}(C)$ is the quotient of $G$ obtained by equating any two braids that differ by a sequence of crossing changes involving pairs of strands whose colors differ by at least two. Thus we can represent an element of $\pi_{1}(C)$ by a braid in $G$ (nonuniquely). We will put the straight strands corresponding to puncture points in whatever position is convenient, and not necessarily on the left.

\subsection{Two homomorphisms}

We now define two group homomorphisms

$$
\begin{gathered}
\rho: B_{\mathbf{c}} \rightarrow\left\{ \pm q^{i / 2} \mid i \in \mathbf{Z}\right\}, \\
\rho^{\prime}: \pi_{1}(C) \rightarrow\left\{ \pm q^{i} \mid i \in \mathbf{Z}\right\} .
\end{gathered}
$$

Each of these maps into the multiplicative group of unit complex numbers.

In the following definitions, a crossing in a braid is called positive if the strand going from the top right to the bottom left passes over the other strand, and negative otherwise. 
The orientations of the strands of colors 0 and $N+1$ play no role in this paper except to determine the orientations on the components of $\widetilde{\beta}$.

Suppose $g$ is an element of $B_{\mathbf{c}}$. Represent $g$ by a braid diagram. To every positive crossing in $g$, associate the monomial

- $q^{N / 2}$ if it involves two strands of the same color, and

- $q^{-(N+1) / 2}$ if it involves two strands of different colors.

To every negative crossing, associate the reciprocal of the term associated to the analogous positive crossing. Let $\rho(g)$ be the product of the monomials associated to the crossings of $g$.

Now suppose $g$ is an element of $\pi_{1}(C)$. Represent $g$ by a braid diagram. To every positive crossing in this braid diagram, associate the term

- $-q^{-1}$ if it involves two strands of the same color,

- $q^{1 / 2}$ if it involves two strands whose colors differ by one, and

- 1 otherwise.

To every negative crossing, associate the reciprocal of the term associated to the analogous positive crossing. Let $\rho^{\prime}(g)$ be the product of the terms associated to the crossings of the braid diagram. The exponent of $q$ in $\rho^{\prime}(g)$ is an integer, since there must be an even number of crossings involving strands whose colors differ by one.

\section{A torus and a ball}

The aim of this section is to define an immersion $\Phi$ from an $m$-dimensional torus to $C$, and an embedding $\Psi$ from an open $m$-ball to $C$. (As usual, $m=N n$.) Until otherwise stated, we assume that $n=1$, and hence that $\mathbf{c}=(0, N+1)$ and $\mathbf{c}^{\prime}=(1,2, \ldots, N)$.

Let $S^{1}$ be the unit circle centered at the origin in the complex plane, and let $T$ be the product of $N$ copies of $S^{1}$. Let $A$ and $B$ be the intersections of $S^{1}$ with the closed upper and lower half planes respectively.

Let $\gamma_{1}, \ldots, \gamma_{N}: S^{1} \rightarrow D$ be figures of eight as shown in Figure 1. Assume $\gamma_{i}$ is parametrized so that $\left.\gamma_{i}\right|_{A}$ is a loop that winds counterclockwise around $p_{1}$, and $\left.\gamma_{i}\right|_{B}$ is a loop that winds clockwise around $p_{2}$. Thus the loops $\gamma_{i}(A)$ are concentric loops around $p_{1}$, and the loops $\gamma_{i}(B)$ are concentric loops around $p_{2}$. We assume that the points $\gamma_{i}(1)$ are all on the real line, and

$$
p_{1}<\gamma_{1}(1)<\cdots<\gamma_{N}(1)<p_{2} .
$$




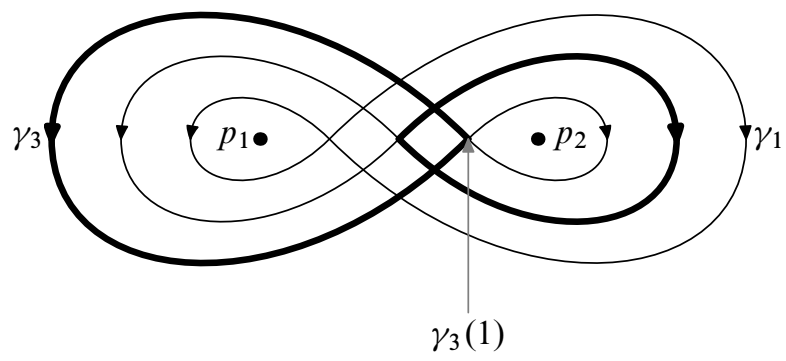

Figure 1: Figures of eight for $N=3$, with $\gamma_{3}(A)$ and $\gamma_{2}(B)$ in bold

\subsection{A special case}

We now define $\Phi: T \rightarrow C$ when $n=1$ and $N=2$. The most difficult part of $\Phi$ is given by the following lemma.

Lemma 3.1 There is an immersion $\Phi_{1}: B \times A \rightarrow C$ such that

$$
\left.\Phi_{1}\right|_{\partial(B \times A)}=\left.\left(\gamma_{1} \times \gamma_{2}\right)\right|_{\partial(B \times A)} .
$$

Proof It suffices to show that the loop $\left.\left(\gamma_{1} \times \gamma_{2}\right)\right|_{\partial(B \times A)}$ is null-homotopic in $C$. This loop is the commutator of the loops $\alpha$ and $\beta$, where $\alpha: A \rightarrow C$ is given by

$$
\alpha(s)=\left(\gamma_{1}(1), \gamma_{2}(s)\right),
$$

and $\beta: B \rightarrow C$ is given by

$$
\beta(s)=\left(\gamma_{1}(s), \gamma_{2}(1)\right) .
$$

We can represent $\alpha$ by a braid as follows:

$$
\alpha=1 / 1
$$

Here, the strands are colored $0,1,2$, and 3 , from left to right. The straight strands on the far left and the far right represent the puncture points.

Recall that the strand of color 2 may pass through the strand of color 0 . Thus $\alpha$ is homotopic relative to endpoints to the loop $\alpha^{\prime}$ represented by the braid as follows:

$$
\alpha^{\prime}=|\%|
$$

Similarly, $\beta$ is homotopic relative to endpoints to $\beta^{\prime}=\left(\alpha^{\prime}\right)^{-1}$. Obviously $\alpha^{\prime}$ and $\beta^{\prime}$ commute, thus completing the proof. 
We can now define $\Phi: T \rightarrow C$ as follows.

$$
\Phi\left(s_{1}, s_{2}\right)= \begin{cases}\Phi_{1}\left(s_{1}, s_{2}\right) & \text { if }\left(s_{1}, s_{2}\right) \in B \times A, \\ \left(\gamma_{1}\left(s_{1}\right), \gamma_{2}\left(s_{2}\right)\right) & \text { otherwise }\end{cases}
$$

This completes the definition of $\Phi$ when $n=1$ and $N=2$. We can choose $\Phi_{1}$ to have some properties that will be useful later.

Lemma 3.2 The function $\Phi_{1}$ in the previous lemma can be chosen so that for every $\left(x_{1}, x_{2}\right)$ in its image,

- $x_{1}$ lies in the closed disk bounded by $\gamma_{1}(B)$,

- $x_{2}$ lies in the closed disk bounded by $\gamma_{2}(A)$, and

- at least one of $x_{1}$ and $x_{2}$ lies in the intersection of these two disks.

Proof Let $C^{\prime}$ be the set of points $\left(x_{1}, x_{2}\right) \in C$ satisfying the three requirements of the lemma. Let $C^{\prime \prime}$ be the set of points $\left(x_{1}, x_{2}\right) \in C^{\prime}$ such that $x_{1}$ and $x_{2}$ both lie in the intersection of the closed disks bounded by $\gamma_{1}(B)$ and $\gamma_{2}(A)$. Let $\alpha, \alpha^{\prime}, \beta$ and $\beta^{\prime}$ be as in the proof of the previous lemma.

Any reasonable choice of homotopy from $\alpha$ to $\alpha^{\prime}$ relative to endpoints will lie in $C^{\prime}$. Further, we can assume that $\alpha^{\prime}$ lies in $C^{\prime \prime}$. Similarly, we can assume that the homotopy from $\beta$ to $\beta^{\prime}$ lies in $C^{\prime}$, and $\beta^{\prime}$ lies in $C^{\prime \prime}$. The commutator of $\alpha^{\prime}$ and $\beta^{\prime}$ is null homotopic as a loop in $C^{\prime \prime}$.

\subsection{General values of $N$}

We now define $\Phi: T \rightarrow C$ when $n=1$ and $N$ is any positive integer. We will use functions

$$
\Phi_{1}, \ldots, \Phi_{N-1}: B \times A \rightarrow D \times D,
$$

similar to $\Phi_{1}$ for the case $N=2$. Specifically,

$$
\left.\Phi_{i}\right|_{\partial(B \times A)}=\left.\left(\gamma_{i} \times \gamma_{i+1}\right)\right|_{\partial(B \times A)},
$$

and for all $\left(x_{i}, x_{i+1}\right)$ in the image of $\Phi_{i}$,

- $x_{i} \neq x_{i+1}$,

- $x_{i}$ lies in the closed disk bounded by $\gamma_{i}(B)$,

- $x_{i+1}$ lies in the closed disk bounded by $\gamma_{i+1}(A)$, and

- at least one of $x_{i}$ and $x_{i+1}$ lies in the intersection of these two disks.

Suppose $\left(s_{1}, \ldots, s_{N}\right) \in T$. For $i=1, \ldots, N$, let $x_{i}$ be as follows. 
- If $s_{i}, s_{i-1} \in A$ then $x_{i}=\gamma_{i}\left(s_{i}\right)$.

- If $s_{i} \in A$ and $s_{i-1} \in B$ then $x_{i}$ is the second coordinate of $\Phi_{i-1}\left(s_{i-1}, s_{i}\right)$.

- If $s_{i}, s_{i+1} \in B$ then $x_{i}=\gamma_{i}\left(s_{i}\right)$.

- If $s_{i} \in B$ and $s_{i+1} \in A$ then $x_{i}$ is the first coordinate of $\Phi_{i}\left(s_{i}, s_{i+1}\right)$.

Here, for convenience, we take $s_{0}$ to be a point in $A \backslash B$ and $s_{N+1}$ to be a point in $B \backslash A$. Let $\Phi\left(s_{1}, \ldots, s_{N}\right)=\left(x_{1}, \ldots, x_{N}\right)$.

We must show that $\Phi$ is a well defined map from $T$ to $C$. First note that if two or more of the conditions apply in the definition of $x_{i}$ then they all give the value $x_{i}=\gamma_{i}\left(s_{i}\right)$. Next note that $x_{1} \neq p_{1}$, since either $x_{1}=\gamma_{1}\left(s_{1}\right)$ or $x_{1}$ is the first coordinate of $\Phi_{1}\left(s_{1}, s_{2}\right)$. Similarly, $x_{N} \neq p_{2}$. It remains to show that $x_{i} \neq x_{i+1}$ for all $i=1, \ldots, N-1$. There are several cases to check.

First, suppose $s_{i} \in A$. Then either $x_{i}=\gamma_{i}\left(s_{i}\right)$ or $x_{i}$ is the second coordinate of $\Phi_{i-1}\left(s_{i-1}, s_{i}\right)$. Also, either $x_{i+1}=\gamma_{i+1}\left(s_{i+1}\right)$ or $x_{i+1}$ is the first coordinate of $\Phi_{i+1}\left(s_{i+1}, s_{i+2}\right)$. In all cases, $x_{i}$ lies in the disk bounded by $\gamma_{i}(A)$, and $x_{i+1}$ does not. Thus $x_{i} \neq x_{i+1}$.

The case $s_{i+1} \in B$ is similar.

Finally, if $s_{i} \in B$ and $s_{i+1} \in A$ then $\left(x_{i}, x_{i+1}\right)=\Phi_{i}\left(s_{i}, s_{i+1}\right)$, so $x_{i} \neq x_{i+1}$. This completes the proof that $\Phi$ is a well defined map from $T$ to $C$. The following lemma gives one of its important properties.

Lemma 3.3 $\rho^{\prime} \circ \Phi_{*}\left(\pi_{1}(T)\right)=\{1\}$.

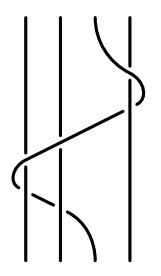

Figure 2: A braid representing $g_{2}$ when $N=2$

Proof For $i=1, \ldots, N$, let $g_{i}: S^{1} \rightarrow T$ be the map $g_{i}(s)=(1, \ldots, 1, s, 1, \ldots, 1)$, where the $s$ occurs in entry number $i$. Then $\Phi \circ g_{i}$ is the loop in $C$ given by

$$
\Phi \circ g_{i}(s)=\left(\gamma_{1}(1), \ldots, \gamma_{i-1}(1), \gamma_{i}(s), \gamma_{i+1}(1), \ldots, \gamma_{N}(1)\right)
$$


Represent this by a mixed braid with $N+2$ strands. Every strand is straight except for the strand with color $i$, which describes a figure of eight. See Figure 2. There are two positive crossings that involve a pair of strands with colors $i$ and $i-1$, and two negative crossings that involve a pair of strands with colors $i$ and $i+1$. Thus $\rho^{\prime}\left(\Phi \circ g_{i}\right)=1$. Since the loops $g_{i}$ generate $\pi_{1}(T)$, this completes the proof.

\subsection{Working with the immersed torus}

Let $T^{\prime}$ be the union of the $N+1$ subsets of $T$ of the form

$$
A \times \cdots \times A \times B \times \cdots \times B
$$

where the product is of some number of copies of $A$ (possibly zero) followed by some number of copies of $B$ (possibly zero). Then the restriction of $\Phi$ to $T^{\prime}$ is simply $\gamma_{1} \times \cdots \times \gamma_{N}$. This makes it fairly easy to work with $\Phi\left(T^{\prime}\right)$.

Now let $X$ be the intersection of the disks bounded by $\gamma_{1}(B)$ and $\gamma_{N}(A)$, and let $C_{X}$ be the set of points in $C$ that include at least one mobile point in $X$. The image under $\Phi$ of any point in $T \backslash T^{\prime}$ involves at least one of the maps $\Phi_{1}, \ldots, \Phi_{N-1}$. Thus $\Phi\left(T \backslash T^{\prime}\right)$ is contained in $C_{X}$. In practice, we can often assume that $X$ is small, and that we can safely ignore $\Phi\left(T \backslash T^{\prime}\right)$.

From now on we will omit any reference to $\Phi$, and treat $T$ as if it were an oriented $N$-dimensional submanifold of $C$.

\subsection{A basepoint}

Choose points $t_{1}, \ldots, t_{N}$ in the disk such that

- $t_{i} \in \gamma_{i}(B)$,

- $t_{i}$ is below the real line, and

- the real parts of $t_{1}, \ldots, t_{N}$ are in increasing order and lie between $\gamma_{N}(1)$ and $p_{2}$.

Let $\mathbf{t}=\left(t_{1}, \ldots, t_{N}\right)$. This will be our basepoint of $T$.

For $i=1, \ldots, N$, let $\tau_{i}: I \rightarrow D$ be a vertical edge from a point on the lower half of $\partial D$ up to $t_{i}$. Let $\tau: I \rightarrow C$ be the path

$$
\tau(s)=\left(\tau_{1}(s), \ldots, \tau_{N}(s)\right) .
$$

Let $\mathbf{x}=\tau(0)$. This will be our basepoint for $C$. Thus $\tau$ is a path from the basepoint $\mathbf{x}$ of $C$ to the basepoint $\mathbf{t}$ of $T$.

Algebraic $8 \mathcal{G}$ Geometric Topology, Volume 7 (2007) 


\subsection{A ball}

Let

$$
S=\left\{\left(s_{1}, \ldots, s_{N}\right) \in \mathbf{R}^{N} \mid 0<s_{1}<\cdots<s_{N}<1\right\} .
$$

This is an open $N$-ball. Let $\gamma: I \rightarrow D$ be the straight edge from $p_{1}$ to $p_{2}$. Let $\Psi: S \rightarrow C$ be the embedding

$$
\Psi\left(s_{1}, \ldots, s_{N}\right)=\left(\gamma\left(s_{1}\right), \ldots, \gamma\left(s_{N}\right)\right) .
$$

From now on we will omit any reference to $\Psi$, and treat $S$ as an oriented $N-$ dimensional submanifold of $C$.

For $i=1, \ldots, N$, let $\zeta_{i}: I \rightarrow D$ be a vertical edge from $x_{i}$ to a point on $\gamma$. Let $\zeta: I \rightarrow C$ be the map

$$
\zeta(s)=\left(\zeta_{1}(s), \ldots, \zeta_{N}(s)\right) .
$$

Let $\mathbf{s}=\zeta(1)$. This will be our basepoint for $S$. Thus $\zeta$ is a path from the basepoint $\mathbf{x}$ of $C$ to the basepoint $\mathbf{s}$ of $S$.

\subsection{General values of $n$}

We now define $T$ and $S$ for any positive integers $n$ and $N$.

Let $C_{1}=C_{(1, \ldots, N)}\left(D_{(0, N+1)}\right)$. This is the configuration space in the case $n=1$. Note that $D_{\mathbf{c}}$ can be obtained by gluing together $n$ (topological) copies of $D_{(0, N+1)}$ side by side. This defines an embedding from the product of $n$ copies of $C_{1}$ into $C$.

Let $T$ be the product of $n$ copies of the immersed $N$-torus in $C_{1}$ as defined in the case $n=1$. Let $\tau$ be the product of $n$ copies of the path in $C_{1}$. This is a path from a basepoint $\mathbf{x}$ of $C$ to a basepoint $\mathbf{t}$ of $T$.

Define an open $m$-ball $S$ and a path $\zeta$ from $\mathbf{x}$ to a basepoint $\mathbf{s}$ of $S$ similarly, by taking a product of $n$ copies of the versions when $n=1$.

\section{Definition of the invariant}

The aim of this section is to define the invariant $Q(\beta)$. We give two equivalent definitions of an intersection pairing $\langle S, \beta(T)\rangle$. The first gives an explicit method of computation, and the second uses a more abstract homological approach. We then define $Q(\beta)$ to be a renormalization of $\langle S, \beta(T)\rangle$.

Algebraic ${ }^{3} \mathcal{G}$ Geometric Topology, Volume 7 (2007) 


\subsection{An intersection pairing}

We can represent $\beta$ by a homeomorphism from $D$ to itself that fixes the boundary pointwise, and preserves the colors of the puncture points. This induces a homeomorphism from $C$ to itself, which we also call $\beta$.

Note that $S$ and $\beta(T)$ are immersed $m$-manifolds in the $(2 m)$-manifold $C$. By applying a small isotopy we can assume that they intersect transversely at a finite number of points. For each such intersection point $\mathbf{y}$, let $\epsilon_{\mathbf{y}}$ be the sign of the intersection at $\mathbf{y}$, and let $\xi_{\mathbf{y}}$ be the composition of the following paths in order:

- $\beta \circ \tau$

- a path in $\beta(T)$ from $\beta(\mathbf{t})$ to $\mathbf{y}$

- a path in $S$ from $\mathbf{y}$ to $\mathbf{S}$

- $\bar{\zeta}$, the path $\zeta$ in the opposite orientation

Note that $\xi_{\mathbf{y}}$ is a closed loop based at $\mathbf{x}$, since $\beta(\mathbf{x})=\mathbf{x}$. Let

$$
\langle S, \beta(T)\rangle=\sum \epsilon_{\mathbf{y}} \rho^{\prime}\left(\xi_{\mathbf{y}}\right),
$$

where the sum is taken over all $\mathbf{y} \in S \cap \beta(T)$.

We now describe how one could use this definition to explicitly compute $\langle S, \beta(T)\rangle$ for a given $\beta$. The computation is complicated, and impractical in all but the simplest examples. However it might provide an aid to understanding, and some aspects of it will be used later in the paper.

We first describe how to recognize a point $\mathbf{y}$ in the intersection of $S$ and $\beta(T)$. Note that $\mathbf{y}$ lies in $S$ if and only if every interval $\left[p_{2 i-1}, p_{2 i}\right]$ contains $N$ of the mobile points of $\mathbf{y}$, having colors $1, \ldots, N$, reading from left to right.

Recall that $T$ is the product of $n$ tori, which we will call $T_{1}, \ldots, T_{n}$. Corresponding to each torus is a small disk $X$ in $D$, as defined in Section 3.3. If necessary, apply an isotopy to $\beta$ so that the images of these disks under $\beta$ are disjoint from the intervals $\left[p_{2 j-1}, p_{2 j}\right]$ used to define $S$.

Suppose $\mathbf{y} \in S$. None of the mobile points in $\mathbf{y}$ lie in the image under $\beta$ of any of the copies of the disk $X$. Thus $\mathbf{y}$ lies on $\beta(T)$ if and only if it lies in the image under $\beta$ of the product of $n$ copies of the set $T^{\prime}$ defined in Section 3.3. In other words, $\mathbf{y}$ lies on $\beta(T)$ if and only if the following conditions hold for each $T_{i}$. Let $\gamma_{1}, \ldots, \gamma_{N}$ be the figures of eight used to define $T_{i}$. Then, for every $j=1, \ldots, N$, $\mathbf{y}$ must include one mobile point of color $j$ on $\beta\left(\gamma_{j}\right)$. This lies in one of the two loops that make 
up $\beta\left(\gamma_{j}\right)$. Taking the corresponding loops for all $j$, we must have the innermost $N_{1}$ loops around one of the puncture points, and the innermost $N_{2}$ loops around the other, for some $N_{1}$ and $N_{2}$ with $N_{1}+N_{2}=N$.

Using the above descriptions, it is possible to list every point $\mathbf{y}$ in the intersection of $S$ and $\beta(T)$. It remains to compute the contribution of each such $\mathbf{y}$ to $\langle S, \beta(T)\rangle$.

First we describe how to find a braid diagram representing the path $\xi_{\mathbf{y}}$ in the case $n=1$. The two strands corresponding to puncture points will always be straight. For $i=1, \ldots, N$, the strand of color $i$ describes a path along $\beta\left(\tau_{i}\right)$ and then along $\beta\left(\gamma_{i}\right)$ to the mobile point that lies on this figure of eight. There is some flexibility in the relative timing of these paths. For example, we can make any choice in which the paths along $\beta\left(\gamma_{i}\right)$ occur in the order $i=1, \ldots, N$.

A crossing in the braid diagram of $\xi_{\mathbf{y}}$ corresponds to a configuration in which two mobile points lie in the same vertical line. Here, we assume $\xi_{\mathbf{y}}$ is in general position. The last half of $\xi_{\mathbf{y}}$, which lies in $S$ and $\zeta$, contributes no crossings to the braid diagram.

The case $n>1$ is basically the same as the case $n=1$. The $2 n$ strands corresponding to puncture points are straight. Each of the remaining $m$ strands describes a path along the copies of $\beta\left(\tau_{i}\right)$ and $\beta\left(\gamma_{i}\right)$ from the appropriate torus $\beta\left(T_{j}\right)$. Within each torus $T_{j}$, perform the paths along $\beta\left(\gamma_{i}\right)$ in the order $i=1, \ldots, N$.

Finally, we describe how to compute the sign $\epsilon_{\mathbf{y}}$. Each mobile point of $\mathbf{y}$ is a point of intersection between some edge from $p_{2 j-1}$ to $p_{2 j}$ and the image under $\beta$ of one of the figures of eight used to define $T$. Determine the sign of this intersection, taking the oriented edge first, and the oriented figure of eight second. Then $\epsilon_{\mathbf{y}}$ is the product of the signs of the intersections at the mobile points of $\mathbf{y}$, multiplied by the sign of the permutation of the mobile points induced by the loop $\xi_{\mathbf{y}}$.

This completes the computation of $\langle S, \beta(T)\rangle$. By Lemma 3.3, $\rho^{\prime}\left(\xi_{\mathbf{y}}\right)$ does not depend on the choice of path in $\beta(T)$. It remains to check that the sum is invariant under isotopy of $\beta$. One could do this by checking invariance under certain moves. However the real reason $\langle S, \beta(T)\rangle$ is well defined is that it computes the homological intersection pairing described below.

\subsection{A homological definition}

We now define some homology modules of $C$.

Let $\mathcal{L}$ be the flat complex line bundle over $C$ with monodromy given by $\rho^{\prime}$. Let $\langle\cdot, \cdot\rangle$ be the sesquilinear inner product on $\mathbf{C}$ given by $\langle x, y\rangle=\bar{x} y$. This inner product is

preserved by the monodromy of $\mathcal{L}$, so it gives a well-defined inner product on the fiber 
of $\mathcal{L}$ at any point. In other words, $\mathcal{L}$ is a Hilbert line bundle. Topologists may prefer to give $\mathbf{C}$ the discrete topology and think of $\mathcal{L}$ as a covering space of $C$. Each fiber of this covering space has the structure of a 1-dimensional Hilbert space, and these structures are locally consistent.

Let $H_{m}(C ; \mathcal{L})$ denote the $m$-dimensional homology of $C$ with local coefficients. For a definition of homology with local coefficients, see, for example, Hatcher [6, Section $3 \mathrm{H}]$. It is similar to singular homology with module coefficients, except that the coefficient of a simplex is a lift of that simplex to $\mathcal{L}$.

Let $H_{m}^{\ell f}(C ; \mathcal{L})$ denote the $m$-dimensional locally finite homology of $C$ with local coefficients (also called Borel-Moore homology). For a definition of locally finite homology, see, for example, Hatcher [6, Exercise 3H.6]. Briefly, the idea is to allow infinite sums of simplices with local coefficients, as long as every compact set in $C$ meets only finitely many simplices.

From now on, all homology modules will be assumed to use coefficients in $\mathcal{L}$. For example, we will write $H_{m}(C)$ to mean $H_{m}(C ; \mathcal{L})$. We also use relative versions of these homology theories. Recall the following basic theorems.

Theorem 4.1 (Poincaré-Lefschetz Duality) $H^{m}(C)$ and $H_{m}^{\ell f}(C, \partial C)$ are isomorphic.

Theorem 4.2 (The Universal Coefficient Theorem) $H^{m}(C)$ and $\operatorname{Hom}\left(H_{m}(C), \mathbf{C}\right)$ are conjugate-isomorphic.

These theorems imply that $H_{m}^{\ell f}(C, \partial C)$ is conjugate-isomorphic to $\operatorname{Hom}\left(H_{m}(C), \mathbf{C}\right)$. Thus there is a sesquilinear pairing

$$
\langle\cdot, \cdot\rangle: H_{m}^{\ell f}(C, \partial C) \times H_{m}(C) \rightarrow \mathbf{C} .
$$

The precise definition of this pairing follows from the more explicit statements of Poincaré-Lefschetz duality and the universal coefficient theorem, which give definitions of the isomorphisms.

Let $\beta_{*}$ be the automorphism of $\pi_{1}(C)$ induced by $\beta$. It is not too hard to show that $\rho^{\prime} \circ \beta_{*}=\rho^{\prime}$. Thus $\beta$ lifts to an action on $\mathcal{L}$. Choose this lift to act as the identity on the fiber over the basepoint $\mathbf{x}$. Thus there are induced actions of $\beta$ on $H_{m}(C)$, $H_{m}(C, \partial C)$ and $H_{m}^{\ell f}(C)$. By abuse of notation, we use $\beta$ to denote every one of these induced actions.

For the rest of this paper, fix an identification of the fiber over $\mathbf{x}$ with $\mathbf{C}$. Let $\tilde{\tau}$ be the lift of $\tau$ to $\mathcal{L}$ starting at the element 1 of the fiber over $\mathbf{x}$. By Lemma 3.3, we can 
lift $T$ to an immersed torus $\widetilde{T}$ in $\mathcal{L}$ such that $\widetilde{T}$ contains $\tilde{\tau}(1)$. This determines an element of $H_{m}(C)$, which we also denote by $T$.

Similarly, $\zeta$ determines a lift $\widetilde{S}$ of $S$ to $\mathcal{L}$. Let $S$ denote the open $m$-ball, the corresponding element of $H_{m}^{\ell f}(C)$, and also the corresponding element of $H_{m}^{\ell f}(C, \partial C)$. Then $\langle S, \beta(T)\rangle$ is the sesquilinear pairing of $S \in H_{m}^{\ell f}(C, \partial C)$ and $\beta(T) \in H_{m}(C)$.

We list some properties of the pairing $\langle\cdot, \cdot\rangle$.

- It is the same as the previous more computational definition.

- It is sesquilinear (conjugate-linear in the first entry and linear in the second).

- It is invariant under the action of $B_{\mathbf{c}}$.

- It has the following symmetry property: if $v_{1}, v_{2} \in H_{m}(C)$ and $v_{1}^{\prime}, v_{2}^{\prime}$ are their

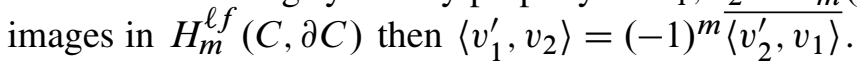

These all follow from standard results in homology theory.

As an aside, note that it might be possible to obtain a unitary representation of $B_{\mathbf{c}}$ with some more work along these lines. Compare the result of Budney [4] that the Lawrence-Krammer representation is negative-definite Hermitian.

\subsection{Definition of the invariant}

We are finally ready to define the invariant $Q(\beta)$. Let

$$
[N+1]=\frac{q^{(N+1) / 2}-q^{-(N+1) / 2}}{q^{1 / 2}-q^{-1 / 2}} .
$$

This is the quantum integer corresponding to $N+1$. Then let

$$
Q(\beta)=\frac{\rho(\beta)}{[N+1] q^{m / 2}}\langle S, \beta(T)\rangle .
$$

The main result of this paper is that $Q(\beta)=P(\tilde{\beta})$.

\section{Height-preserving isotopy}

For all $i=1, \ldots, n-1$, let $\sigma_{i}^{\prime}=\sigma_{2 i} \sigma_{2 i+1} \sigma_{2 i-1} \sigma_{2 i}$. The aim of this section is to prove the following.

Lemma 5.1 $Q\left(\sigma_{1}^{2} \beta\right)=Q\left(\beta \sigma_{1}^{2}\right)=Q\left(\sigma_{i}^{\prime} \beta\right)=Q\left(\beta \sigma_{i}^{\prime}\right)=Q(\beta)$. 
Assume the plat closure $\widetilde{\beta}$ is defined so that all maxima are at the same height and all minima are at the same height. Then the above lemma is equivalent to the statement that $Q(\beta)$ is invariant under height preserving isotopy of $\widetilde{\beta}$. We will not use this formulation, but mention it by way of motivation.

Claim $Q\left(\sigma_{1}^{2} \beta\right)=Q(\beta)$.

Proof We have

$$
\rho\left(\sigma_{1}^{2} \beta\right)=q^{-(N+1)} \rho(\beta) .
$$

By this and the properties of the sesquilinear pairing, it suffices to show

$$
\sigma_{1}^{2} S=q^{N+1} S
$$

We can choose the function $\sigma_{1}^{2}$ to act as the identity on the subset $S$ of $C$. It remains to show that $\sigma_{1}^{2}$ acts as multiplication by $q^{N+1}$ on the fiber over $\mathbf{s}$.

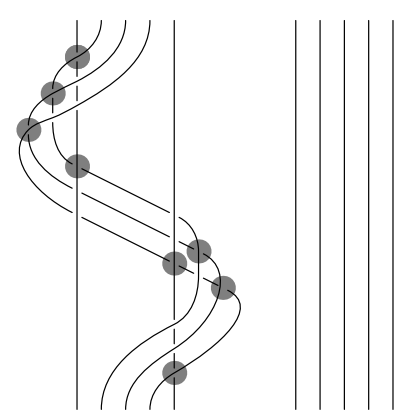

Figure 3: $\left(\sigma_{1}^{2} \zeta\right) \cdot(\bar{\zeta})$, with relevant crossings shaded

Let $\xi$ be the concatenation of the paths $\sigma_{1}^{2} \zeta$ and $\bar{\zeta}$. This is represented by a braid in which strands of colors $1, \ldots, N$ make a positive full twist around two with colors 0 and $N+1$. For example, Figure 3 shows the case where $n=2$ and $N=3$. The relevant crossings are those between strands of neighboring colors. There are $N+1$ pairs of strands that have neighboring colors, and each such pair makes two positive crossings. Thus

$$
\rho^{\prime}(\xi)=\left(q^{1 / 2}\right)^{2 N+2}=q^{N+1} .
$$

Thus $\sigma_{1}^{2}(S)=q^{N+1} S$, as required.

Claim $Q\left(\sigma_{i}^{\prime} \beta\right)=Q(\beta)$. 
Proof We have

$$
\rho\left(\sigma_{i}^{\prime} \beta\right)=q^{-1} \rho(\beta) .
$$

By this and the properties of the sesquilinear pairing, it suffices to show

$$
\sigma_{i}^{\prime} S=q S .
$$

We can choose the function $\sigma_{i}^{\prime}$ to preserve $S$ as a subset of $C$. However $\sigma_{i}^{\prime}$ reverses the order of two of the $N$-balls used to define $S$. This represents a change of orientation if and only if $N$ is odd. It remains to show that $\sigma_{i}^{\prime}$ acts as multiplication by $(-1)^{N} q$ on the fiber over $\mathbf{s}$.

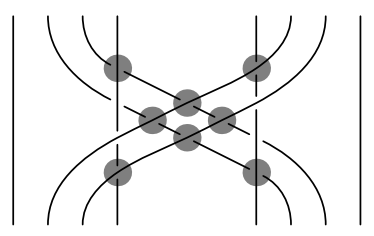

Figure 4: $\left(\sigma_{1}^{\prime} \zeta\right) \cdot(\bar{\zeta})$, with relevant crossings shaded

Let $\xi_{i}$ be the concatenation of the paths $\sigma_{i}^{\prime} \zeta$ and $\bar{\zeta}$. This is represented by a braid in which two collections of $N$ parallel strands of colors $1, \ldots, N$ form a large letter $X$ enclosing two strands of colors 0 and $N+1$. For example, Figure 4 shows the case where $n=2$ and $N=2$. There are a total of $4 N$ crossings that involve strands corresponding to puncture points. Of these, there are $N$ crossings between strands whose colors differ by 1 . There are a total of $N^{2}$ crossings that do not involve a puncture point. Of these, there are $N$ crossings between strands of the same color, and $2(N-1)$ crossings between strands whose colors differ by 1 . Altogether we have

$$
\rho^{\prime}\left(\xi_{i}\right)=\left(-q^{-1}\right)^{N}\left(q^{1 / 2}\right)^{2 N+2}=(-1)^{N} q .
$$

Thus $\sigma_{i}^{\prime}(S)=(-1)^{N} q S$, as required.

It remains to show that $Q\left(\beta \sigma_{1}^{2}\right)=Q(\beta)$ and $Q\left(\beta \sigma_{i}^{\prime}\right)=Q(\beta)$. It suffices to show that $\sigma_{1}^{2} T=q^{N+1} T$ and $\sigma_{i}^{\prime}(T)=q T$. The proof of these identities is the same as the proof of the analogous identities for $S$ given in the previous two claims. This completes the proof of the lemma.

\section{Barcodes}

Before we prove the invariance of $Q(\beta)$ under other moves, we will look more closely at $H_{m}^{\ell f}(C)$ and $H_{m}(C, \partial C)$. In the process, we will introduce a useful tool I call a barcode. 


\subsection{A basis for locally finite homology}

Let $C_{\mathbf{R}}$ be the set of points in $C$ that are configurations of points on the real line in $D$.

Lemma 6.1 The map $H_{m}^{\ell f}\left(C_{\mathbf{R}}\right) \rightarrow H_{m}^{\ell f}(C)$ induced by inclusion is an isomorphism.

For the details of the proof, see Lemma 3.1 of [2]. The idea is to vertically "squash" configurations of points in the disk to configurations of points in the real line. The only difficulty is that a configuration may involve two points (either two mobile points or a mobile point and a puncture point) that lie in the same vertical line, but whose colors differ by at most one. Such a configuration would be "sent to infinity" as it was squashed to the real line. Since we are using locally finite homology, this does not pose a serious problem.

We now enumerate the components of $C_{\mathbf{R}}$.

Definition 6.2 A code sequence is a permutation of the sequence $\mathbf{c c}^{\prime}$ that contains $\mathbf{c}$ as a subsequence.

Suppose $S^{\prime}$ is a connected component of $C_{\mathbf{R}}$. Choose a point $\mathbf{y}=\left(y_{1}, \ldots, y_{m}\right)$ in $S^{\prime}$ such that $y_{1}, \ldots, y_{m}$ are distinct from each other and from the puncture points. Let $\mathbf{c}^{\prime \prime}=\left(c_{1}^{\prime \prime}, \ldots, c_{m+k}^{\prime \prime}\right)$ be the sequence of colors of mobile points and puncture points, reading from left to right on the real line. Then $\mathbf{c}^{\prime \prime}$ is a code sequence. We say $\mathbf{c}^{\prime \prime}$ represents $S^{\prime}$ (nonuniquely).

Suppose $i$ is such that $\left|c_{i}^{\prime \prime}-c_{i+1}^{\prime \prime}\right| \geq 2$ and at least one of $c_{i}^{\prime \prime}$ and $c_{i+1}^{\prime \prime}$ is in $\{1, \ldots, N\}$. Then we can exchange $c_{i}^{\prime \prime}$ and $c_{i+1}^{\prime \prime}$ in $\mathbf{c}^{\prime \prime}$ without altering the connected component of $C_{\mathbf{R}}$ it represents. This corresponds to moving a mobile point through another mobile point or a puncture, provided their colors permit this. We say two code sequences are equivalent if they are related by a sequence of such transpositions. The equivalence classes of code sequences enumerate the connected components of $C_{\mathbf{R}}$.

Definition 6.3 A code sequence is trivial if it is equivalent to a code sequence whose first or last entry lies in $\{1, \ldots, N\}$.

Suppose $S^{\prime}$ is the connected component of $C_{\mathbf{R}}$ corresponding to a code sequence $\mathbf{c}^{\prime \prime}$. If $\mathbf{c}^{\prime \prime}$ is trivial then $S^{\prime}$ contains a point $\left(y_{1}, \ldots, y_{m}\right)$ such that $y_{1}$ or $y_{m}$ lies on $\partial D$. In this case, $S^{\prime}$ is homeomorphic to the closed upper half space in $\mathbf{R}^{m}$, so $H_{m}^{\ell f}\left(S^{\prime}\right)=0$. If $\mathbf{c}^{\prime \prime}$ is not trivial then every point in $S^{\prime}$ is a configuration of points between $p_{1}$ and $p_{2 n}$. In this case, $S^{\prime}$ is homeomorphic to an $\mathbf{R}^{m}$, so $H_{m}^{\ell f}\left(S^{\prime}\right)=\mathbf{C}$. 
To those more familiar with ordinary homology, it may seem strange that $\mathbf{R}^{m}$ has nontrivial locally finite homology, but the closed open half space does not. One way to think of locally finite homology is as homology relative to the complement of a large compact set. (To be precise, one should take an inverse limit.) For either $\mathbf{R}^{m}$ or the closed upper half space, we can take homology relative to the complement of a large closed ball. However in the case of the closed upper half space, this closed ball includes part of the boundary of the space.

For every nontrivial code sequence $\mathbf{c}^{\prime \prime}$, choose a nonzero element of $H_{m}^{\ell f}\left(S^{\prime}\right)$, where $S^{\prime}$ is the corresponding component of $C_{\mathbf{R}}$. By Lemma 6.1, this gives a basis for $H_{m}^{\ell f}(C)$. To define this basis precisely, we would need to specify an orientation and a lift to $\mathcal{L}$ for every component of $C_{\mathbf{R}}$. In practice, it often suffices to specify an element of $H_{m}^{\ell f}(C)$ up to multiplication by a nonzero scalar.

\subsection{A basis for homology relative to boundary}

Let

$$
\langle\cdot, \cdot\rangle^{\prime}: H_{m}^{\ell f}(C) \times H_{m}(C, \partial C) \rightarrow \mathbf{C}
$$

be the nondegenerate sesquilinear pairing defined using the more general version of Poincaré-Lefschetz Duality. We define a basis of $H_{m}(C, \partial C)$ that is dual to our basis of $H_{m}^{\ell f}(C)$ with respect to this pairing.

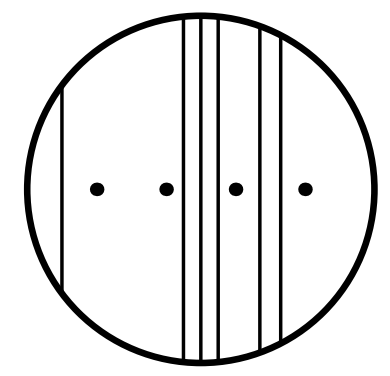

Figure 5: An example of edges used to define a barcode

Let $E_{1}, \ldots, E_{m}$ be vertical edges in $D$ that are disjoint from each other and from the puncture points, and have endpoints on $\partial D$. See, for example, Figure 5. Associate a color to each edge such that the colors of the edges are some permutation of $\mathbf{c}^{\prime}$. Let $\mathbf{c}^{\prime \prime}$ be the sequence of colors of vertical edges or puncture points, reading from left to right. This is a code sequence.

Let $Z$ be the set of configurations that consist of one point on each edge $E_{i}$, having the same color as $E_{i}$. This is a properly embedded closed $m$-ball in $C$. Choose an 
oriented lift of $Z$ to $\mathcal{L}$ that is not the lift to the zero section. This represents an element of $H_{m}(C, \partial C)$. By abuse of notation, we will use $Z$ to denote both the $m$-ball in $C$ and a corresponding element of $H_{m}(C, \partial C)$, and call either of these the barcode corresponding to the code sequence $\mathbf{c}^{\prime \prime}$.

Two equivalent code sequences will give rise to the same barcode in $H_{m}(C, \partial C)$, up to the choices of orientation and lift. If $\mathbf{c}^{\prime \prime}$ is trivial then any barcode corresponding to $\mathbf{c}^{\prime \prime}$ is the zero element of $H_{m}(C, \partial C)$. For each equivalence class of nontrivial code sequences, choose a corresponding barcode in $H_{m}(C, \partial C)$. I claim that these form a basis for $H_{m}(C, \partial C)$.

Suppose $S^{\prime}$ is a component of $C_{\mathbf{R}}$ and $Z$ is a barcode. If $S^{\prime}$ and $Z$ correspond to the same nontrivial code sequence then they intersect at one point, so we can choose our lifts and orientations so that $\left\langle S^{\prime}, Z\right\rangle^{\prime}=1$. On the other hand, if $S^{\prime}$ and $Z$ correspond to different nontrivial code sequences then they do not intersect, so $\left\langle S^{\prime}, Z\right\rangle^{\prime}=0$. Thus we have a basis of $H_{m}(C, \partial C)$ that is dual to our basis for $H_{m}^{\ell f}(C)$.

\subsection{Images of the torus}

Throughout this subsection, unless otherwise stated, we assume $n=1$. We now compute the image of $T$ in $H_{m}^{\ell f}(C)$, and also in $H_{m}(C, \partial C)$.

The unique nontrivial code sequence is $(0,1, \ldots, N+1)$. Let $Z$ be the corresponding barcode. We specify an orientation and lift of $Z$ as follows. Take $Z$ to be the product of edges of colors $1, \ldots, N$ in order, with each edge oriented upwards. We can assume that $Z$ contains the basepoint $\mathbf{x}$. Choose the lift of $Z$ to $\mathcal{L}$ that contains the point 1 in the fiber over $\mathbf{x}$. Note that $\langle S, Z\rangle^{\prime}=1$.

Lemma 6.4 For any $n \geq 1$, the image of $T$ in $H_{m}^{\ell f}(C)$ is $(q-1)^{m} S$.

Proof By the construction of $T$, it suffices to prove this lemma in the case $n=1$.

There is only one nontrivial code sequence, so $T$ is some scalar multiple of $S$ in $H_{m}^{\ell f}(C)$. Let $Z$ be as above. It remains to show that

$$
\langle T, Z\rangle^{\prime}=\overline{(q-1)}^{N} \text {. }
$$

This is equivalent to

$$
\langle Z, T\rangle=(1-q)^{N}
$$

Let $\gamma_{1}, \ldots, \gamma_{N}: S^{1} \rightarrow D$ be the figures of eight used to define $T$, and let $E_{1}, \ldots, E_{N}$ be the edges used to define $Z$. See Figure 6 . For each $i=1, \ldots, N, \gamma_{i}$ intersects $E_{i}$ 


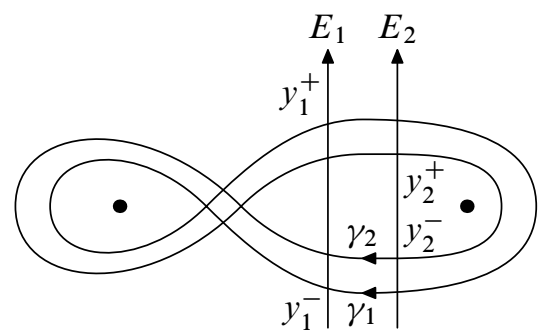

Figure 6: $Z$ and $T$ in the case $N=2$

at two points. Call these points $y_{i}^{+}$and $y_{i}^{-}$, where $y_{i}^{+}$is above $y_{i}^{-}$. Then $Z$ and $T$ intersect at the $2^{N}$ points of the form

$$
\left(y_{1}^{ \pm}, \ldots, y_{N}^{ \pm}\right) .
$$

Each such point $\mathbf{y}$ contributes a monomial $\pm q^{k}$ to $\langle Z, T\rangle$.

Our basepoint of $T$ is given by

$$
\mathbf{t}=\left(y_{1}^{-}, \ldots, y_{N}^{-}\right) .
$$

The orientation of the intersection of $E_{i}$ and $\gamma_{i}$ at $y_{i}^{-}$is positive. Thus $\mathbf{t}$ contributes 1 to $\langle Z, T\rangle$.

Now suppose $\mathbf{y}$ and $\mathbf{y}^{\prime}$ are two points of intersection between $Z$ and $T$ that differ only at the mobile point of color $i$, where $\mathbf{y}$ has $y_{i}^{-}$and $\mathbf{y}^{\prime}$ had $y_{i}^{+}$. Let $\xi$ be the loop in $C$ that follows a path in $T$ from $\mathbf{y}$ to $\mathbf{y}^{\prime}$, and then follows a path in $Z$ back to $\mathbf{y}^{\prime}$. This can be represented by a braid in which all strands are straight except the strand of color $i$, which makes a positive full twist around the strands of color $i+1, \ldots, N+1$. Thus $\rho^{\prime}(\xi)=q$. Also note that the orientation of the intersection at $\mathbf{y}^{\prime}$ is the opposite of that at $\mathbf{y}$. Thus if $\mathbf{y}$ contributes $\pm q^{k}$ to $\langle Z, T\rangle^{\prime}$ then $\mathbf{y}^{\prime}$ contributes $\mp q^{k+1}$.

Summing the contributions of the $2^{N}$ points in $T \cap Z$ we obtain

$$
\langle Z, T\rangle=(1-q)^{N},
$$

as required.

Lemma 6.5 If $n=1$ and $Z$ is as above then the image of $T$ in $H_{m}(C, \partial C)$ is $\left(1+q+\cdots+q^{N}\right) Z$.

Proof Recall that $\langle S, Z\rangle^{\prime}=1$. Thus it suffices to prove the identity

$$
\langle S, T\rangle=1+q+\cdots+q^{N} .
$$




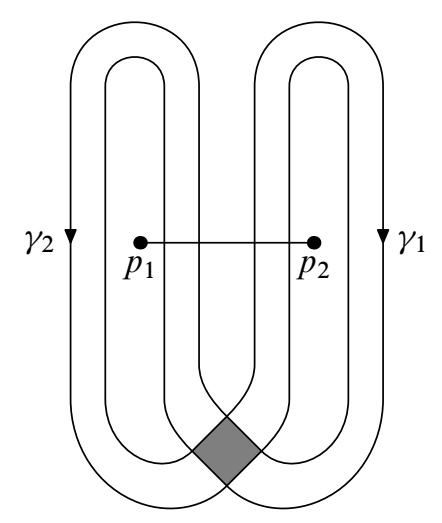

Figure 7: A stretched $T$ for $N=2$, with $X$ shaded

Let $\gamma_{1}, \ldots, \gamma_{N}$ be the figures of eight used to define $T$. Isotope $T$ so that the disk $X$, as defined in Section 3.3, is below the interval $\left[p_{1}, p_{2}\right]$. See Figure 7. Now each $\gamma_{i}$ intersects the interval $\left[p_{1}, p_{2}\right]$ at two points $a_{i}$ and $b_{i}$, where $a_{i}$ is to the left of $b_{i}$. Thus the points $a_{1}, \ldots, a_{N}, b_{1}, \ldots, b_{N}$ are in order from left to right.

For $i=0,1, \ldots, N$, let

$$
\mathbf{y}_{i}=\left(a_{1}, \ldots, a_{i}, b_{i+1}, \ldots, b_{N}\right) .
$$

Then $\mathbf{y}_{0}, \ldots, \mathbf{y}_{N}$ are the points of intersection between $S$ and $T$. Each of these contributes a monomial $\pm q^{k}$ to $\langle S, T\rangle$.

The sign of the intersection of $S$ and $T$ at $\mathbf{y}_{i}$ is positive for all $i=0, \ldots, N$. It is not hard to see that $\mathbf{y}_{N}$ contributes +1 to $\langle S, T\rangle$. We now show that $\mathbf{y}_{i}$ contributes $q^{N-i}$, for all $i=0, \ldots, N$. Let $\xi$ be a loop in $C$ that follows a path in $T$ from $\mathbf{y}_{i}$ to $\mathbf{y}_{i-1}$, and then follows a path in $S$ back to $\mathbf{y}_{i}$. We can choose $\xi$ to be the loop where all mobile points remain stationary except for the point of color $i$, which starts at $a_{i}$, follows $\gamma_{i}$ around $p_{2}$ to $b_{i}$, and then moves horizontally back to $a_{i}$. The point of color $i$ makes a full loop in the positive direction around a point of color $i+1$, Thus $\rho^{\prime}(\xi)=q$, so the contribution of $\mathbf{y}_{i-1}$ is $q$ times the contribution of $\mathbf{y}_{i}$. Summing the contributions of $\mathbf{y}_{i}$ for all $i$ gives the desired identity.

\section{A partial barcode}

The aim of this section is to prove a certain identity in $H_{m}^{\ell f}(C, \partial C)$, which will show that $S$ can be, in some sense, partially converted into a barcode. 
Recall that $S$ was defined to be the product of $n$ copies of an $N$-dimensional ball. Call these $N$-balls $S_{1}, \ldots, S_{n}$. Let $Z$ be the nontrivial barcode for the case $n=1$, as defined in Section 6.3. Let $Z_{i}$ be the product

$$
Z_{i}=S_{1} \times \cdots \times S_{i-1} \times Z \times S_{i+1} \times \cdots \times S_{n} .
$$

The basepoint $\mathbf{s}$ lies in $Z_{i}$, so the path $\zeta$ determines a lift of $Z_{i}$ to $\mathcal{L}$. We obtain an element of $H_{m}^{\ell f}(C, \partial C)$, which we also call $Z_{i}$. The aim of this section is to prove the following.

Lemma 7.1 $(q-1)^{N} S=\left(1+q+\cdots+q^{N}\right) Z_{i}$ in $H_{m}^{\ell f}(C, \partial C)$.

Take $n=1$ until otherwise stated. By Lemma $6.4, T=(q-1)^{N} S$. It remains to show that

$$
T=\left(1+q+\cdots+q^{N}\right) Z_{1} .
$$

But this is immediate from Lemma 6.5. We now describe a more direct approach to proving this identity, and what the result of this approach must be, given that we already know the identity holds.

First, vertically "stretch" $T$, as suggested by Figure 7. Continue this stretching process and use an excision argument to obtain a disjoint union of barcodes. One of these must be $Z_{1}$, with the desired coefficient. Any other barcode must correspond to a trivial code sequence. Such a barcode represents zero in $H_{m}^{\ell f}(C, \partial C)$, since one of the vertical edges can be slid to the boundary of the disk.

We can apply most of this argument to the case $n>1$. The only difficulty is that the $N$-balls $S_{1}, \ldots, S_{i-1}$ and $S_{i+1}, \ldots, S_{n}$ prevent us from simply sliding a vertical edge to the boundary of the disk. To overcome this problem, we prove a claim that will imply that each such $N$-ball is in some sense "transparent" to any other mobile point. We need to make some definitions before we can state the claim precisely.

We continue with the assumption $n=1$, but introduce a configuration space with one extra mobile point. Fix any $j=1, \ldots, N$. Let $\mathbf{c}^{\prime} j$ denote the sequence $(1,2, \ldots, N, j)$. Let $C^{\prime}$ be the configuration space

$$
C^{\prime}=C_{\mathbf{c}^{\prime} j}\left(D_{\mathbf{c}}\right) .
$$

Let $S^{\prime}$ be the product of the usual $N$-ball in $C$ and a circle of color $j$ around the interval $\left[p_{1}, p_{2}\right]$. This is an $(N+1)$-dimensional submanifold of $C^{\prime}$.

Let $g$ be the generator of $\pi_{1}\left(S^{\prime}\right)$. Then $g$ can be represented by a braid with strands of colors $0,1, \ldots, N+1$ that are straight, and another strand of color $j$ that makes a positive full twist around all of the other strands. This strand makes two crossings 
with another strand of color $j$, and four crossings with the two strands of colors $j \pm 1$. Thus

$$
\rho^{\prime}(g)=\left(-q^{-1}\right)^{2}\left(q^{1 / 2}\right)^{4}=1 .
$$

Thus we can lift of $S^{\prime}$ to $\mathcal{L}$. This represents an element of $H_{m}^{\ell f}(C)$, which we also call $S^{\prime}$.

Claim $S^{\prime}=0$ in $H_{m}^{\ell f}\left(C^{\prime}\right)$.

Proof First consider the case $N=1$. Then $S^{\prime}$ is simply the product of an edge $\gamma$ between the two puncture points and a circle $\delta$ around $\gamma$.

The only nontrivial code sequence is $(0,1,1,2)$. Let $Z$ be the corresponding barcode. It suffices to show that

$$
\left\langle S^{\prime}, Z\right\rangle^{\prime}=0 \text {. }
$$

Let $E$ and $E^{\prime}$ be properly embedded vertical edges passing between the puncture points, where $E^{\prime}$ is to the right of $E$. As a closed 2-ball, $Z$ is the product of $E$ and $E^{\prime}$, both having color 1 .

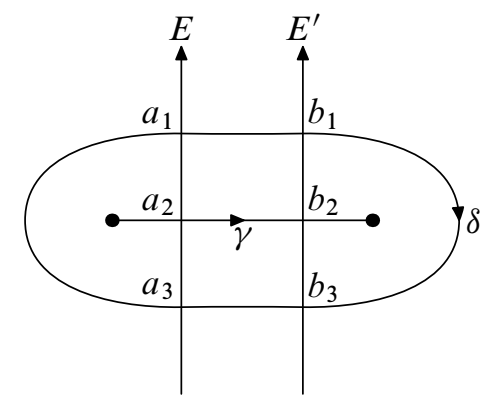

Figure 8: $S^{\prime}$ and $Z$ in the case $N=1$

Let $a_{1}, a_{2}$ and $a_{3}$ be the points of intersection between $E$ and $\gamma \cup \delta$, reading from top to bottom. Let $b_{1}, b_{2}$, and $b_{3}$ be the analogous points of intersection between $E^{\prime}$ and $\gamma \cup \delta$. See Figure 8. There are four points of intersection between $S^{\prime}$ and $Z$ :

- $\mathbf{y}_{1}=\left(a_{2}, b_{1}\right)$

- $\mathbf{y}_{2}=\left(a_{2}, b_{3}\right)$

- $\mathbf{y}_{3}=\left(a_{1}, b_{2}\right)$

- $\mathbf{y}_{4}=\left(a_{3}, b_{2}\right)$

Algebraic $8 \mathcal{G}$ Geometric Topology, Volume 7 (2007) 
Each of these contributes a monomial $\pm q^{k}$ to $\left\langle S^{\prime}, Z\right\rangle^{\prime}$. Assume the orientations and lifts to $\mathcal{L}$ were chosen so that $\mathbf{y}_{1}$ contributes +1 .

For $i=2,3,4$, let $\xi_{i}$ be a loop that follows a path in $Z$ from $\mathbf{y}_{1}$ to $\mathbf{y}_{i}$, and then follows a path in $S^{\prime}$ back to $\mathbf{y}_{1}$. These are represented by the braids

$$
\xi_{2}=|| \frac{1}{1}, \quad \xi_{3}=\left|\gamma^{\prime}\right|, \quad \xi_{4}=|-|^{\prime} \mid
$$

where the strands have colors $0,1,1,2$, reading from left to right. Thus we have the following:

- $\rho^{\prime}\left(\xi_{2}\right)=\left(q^{1 / 2}\right)^{2}=q$

- $\rho^{\prime}\left(\xi_{3}\right)=\left(-q^{-1}\right)^{-1}=-q$

- $\rho^{\prime}\left(\xi_{4}\right)=\left(-q^{-1}\right)^{-1}\left(q^{1 / 2}\right)^{-2}=-1$

For $i=2,3,4$, let $\epsilon_{i}$ be the sign of the intersection of $S^{\prime}$ and $Z$ at $\mathbf{y}_{i}$. For $j=1,2,3$, the intersections of the relevant edges at $a_{j}$ and $b_{j}$ have the same sign. The intersections at $a_{1}$ and $a_{3}$ have opposite signs. The loops $\xi_{3}$ and $\xi_{4}$ transpose the two mobile points, but $\xi_{2}$ does not. Combining these facts, we obtain $\epsilon_{2}=\epsilon_{3}=-1$ and $\epsilon_{4}=1$. Thus

$$
\left\langle S^{\prime}, Z\right\rangle^{\prime}=1+\epsilon_{2} \rho^{\prime}\left(\xi_{2}\right)+\epsilon_{3} \rho^{\prime}\left(\xi_{3}\right)+\epsilon_{4} \rho^{\prime}\left(\xi_{4}\right)=0 .
$$

Now consider the case $N>1$. The only nontrivial code sequence is

$$
(0,1, \ldots, j-1, j, j, j+1, \ldots, N, N+1) .
$$

Let $Z$ be the corresponding barcode. We must show that

$$
\left\langle S^{\prime}, Z\right\rangle^{\prime}=0
$$

As a closed $m$-ball, $Z$ is a product of vertical edges

$$
E_{1}, \ldots, E_{j-1}, E_{j}, E_{j}^{\prime}, E_{j+1}, \ldots, E_{N}
$$

Here, $E_{k}$ has color $k$ for $k=1, \ldots, N$, and $E_{j}^{\prime}$ has color $j$. Let $y_{k}$ be the point of intersection between $E_{k}$ and the interval $\left[p_{1}, p_{2}\right]$. Any point of intersection between $S^{\prime}$ and $Z$ must include the mobile points $y_{k}$ of color $k$ for every $k \neq j$. These points play no important role since they remain the same throughout the proof. The rest of the computation proceeds exactly as in the case $N=1$.

This completes the proof of the claim, and hence of the lemma. 


\section{Bridge-preserving isotopy}

We use the notation

$$
\sigma_{2112}=\sigma_{2} \sigma_{1}^{2} \sigma_{2}
$$

The aim of this section is to prove the following.

Lemma 8.1 $Q\left(\sigma_{2112} \beta\right)=Q\left(\beta \sigma_{2112}\right)=Q(\beta)$.

Combined with Lemma 5.1, this implies that $Q(\beta)$ is invariant under any isotopy of $\tilde{\beta}$ through links that are in bridge position.

Claim $Q\left(\sigma_{2112} \beta\right)=Q(\beta)$.

Proof We have

$$
\rho\left(\sigma_{2112} \beta\right)=q^{-1} \rho(\beta) .
$$

By this and the properties of the sesquilinear pairing, it suffices to show that the identity

$$
\sigma_{2112} S=q S
$$

holds in $H_{m}^{\ell f}(C, \partial C)$.

Let $Z_{2}$ be as defined in Section 7. By Lemma 7.1, it suffices to show that $\sigma_{2112} Z_{2}=$ $q Z_{2}$ in $H_{m}^{\ell f}(C, \partial C)$. We can choose the function $\sigma_{2112}$ to act as the identity on the subset $Z_{2}$ of $C$. It remains to show that $\sigma_{2112}$ acts as multiplication by $q$ on the fiber over $\mathbf{s}$.

Let $\xi$ be the concatenation of the paths $\sigma_{2112} \zeta$ and $\bar{\zeta}$. We can represent $\xi$ by a braid in which strands of color $1, \ldots, N$ wind in parallel around a strand of color 0 . Thus $\rho^{\prime}(\xi)=q$. Thus $\sigma_{2112} Z_{2}=q Z_{2}$, as required.

It remains to show that $Q\left(\beta \sigma_{2112}\right)=Q(\beta)$. It suffices to prove the following.

Claim $Q\left(\beta^{-1}\right)=\overline{Q(\beta)}$.

Proof We have the following identities:

- $q^{m / 2}=q^{m} \overline{\left(q^{m / 2}\right)}$

- $[N+1]=\overline{[N+1]}$

- $\rho\left(\beta^{-1}\right)=\overline{\rho(\beta)}$ 
By the definition of $Q(\beta)$, it remains to show that

$$
\left\langle S, \beta^{-1}(T)\right\rangle=q^{m} \overline{\langle S, \beta(T)\rangle} .
$$

By Lemma 6.4 and the properties of the sesquilinear pairing, this is equivalent to

$$
\langle\beta(T), T\rangle=(-1)^{m} \overline{\langle T, \beta(T)\rangle} .
$$

This follows from the symmetry property of the pairing.

\section{Markov-Birman stabilization}

Recall that $\mathbf{c}=(0, N+1,0, N+1, \ldots, 0, N+1)$. Let $\mathbf{c}_{1}=(0, N+1)$, and let $\mathbf{c c}_{1}$ denote the concatenation of these sequences. This is the sequence of colors of puncture points when we replace $n$ with $n+1$. Let

$$
\iota: B_{\mathbf{c}} \rightarrow B_{\mathbf{c c}_{1}}
$$

be the obvious inclusion map. The Markov-Birman stabilization of $\beta$ is the braid

$$
\beta^{\prime}=\left(\sigma_{2 n+1}^{-1} \sigma_{2 n} \sigma_{2 n+1}\right) \iota(\beta) .
$$

The aim of this section is to prove the following.

Lemma 9.1 If $\beta^{\prime}$ is the Markov-Birman stabilization of $\beta$ then $Q\left(\beta^{\prime}\right)=Q(\beta)$.

Combined with Lemma 5.1 and Lemma 8.1, this implies that $Q(\beta)$ is an invariant of the oriented knot or link $\tilde{\beta}$.

Let $D^{\prime}$ be the $k+2$ times punctured disk $D_{\mathbf{c c}_{1}}$. Let $\mathbf{c}_{1}^{\prime}=(1, \ldots, N)$, and let $\mathbf{c}^{\prime} \mathbf{c}_{1}^{\prime}$ denote the concatenation of these sequences. Let $C^{\prime}$ be the configuration space

$$
C^{\prime}=C_{\mathbf{c}^{\prime} \mathbf{c}_{1}^{\prime}}\left(D^{\prime}\right) \text {. }
$$

Let $S^{\prime}$ and $T^{\prime}$ be the obvious $m^{\prime}$-ball and $m^{\prime}$-torus in $C^{\prime}$.

We have the identity

$$
\rho\left(\beta^{\prime}\right)=q^{N / 2}(\rho(\beta)) .
$$

Due to the coefficients in the definitions of $Q(\beta)$ and $Q\left(\beta^{\prime}\right)$, it now suffices to show

$$
\left\langle S^{\prime}, \beta^{\prime}\left(T^{\prime}\right)\right\rangle=\langle S, \beta(T)\rangle .
$$

Let $Z_{n}$ be the subset of $C$ as defined in Section 7. Let $Z_{n}^{\prime}$ be the subset of $C^{\prime}$ defined similarly, namely by replacing the second to rightmost $N$-ball of $S^{\prime}$ with a barcode. By Lemma 7.1, Equation (1) is equivalent to

$$
\left\langle Z_{n}^{\prime}, \beta^{\prime}\left(T^{\prime}\right)\right\rangle=\left\langle Z_{n}, \beta(T)\right\rangle .
$$

Algebraic $8 \mathcal{G}$ Geometric Topology, Volume 7 (2007) 
This, in turn, is equivalent to

$$
\left\langle\sigma\left(Z_{n}^{\prime}\right), \iota(\beta)\left(T^{\prime}\right)\right\rangle=\left\langle Z_{n}, \beta(T)\right\rangle,
$$

where

$$
\sigma=\sigma_{2 n+1}^{-1} \sigma_{2 n}^{-1} \sigma_{2 n+1} .
$$

First let us look at $\sigma\left(Z_{n}^{\prime}\right)$. Let $D_{3}$ be the three times punctured disk consisting of points in $D^{\prime}$ to the right of a vertical line between $p_{2 n-1}$ and $p_{2 n}$. There is an embedding

$$
C_{\mathbf{c}^{\prime}}\left(D^{\prime} \backslash D_{3}\right) \times C_{\mathbf{c}_{1}^{\prime}}\left(D_{3}\right) \rightarrow C^{\prime} .
$$

We can assume that $Z_{n}$ lies in $C_{\mathbf{c}^{\prime}}\left(D^{\prime} \backslash D_{3}\right)$ and

$$
Z_{n}^{\prime}=Z_{n} \times S_{n+1},
$$

where $S_{n+1}$ is the obvious $N$-ball in $C_{\mathbf{c}_{1}^{\prime}}\left(D_{3}\right)$. Thus

$$
\sigma\left(Z_{n}^{\prime}\right)=Z_{n} \times \sigma\left(S_{n+1}\right) .
$$

Next we look at $\iota(\beta)\left(T^{\prime}\right)$. Let $D_{2}$ be the twice punctured disk consisting of points in $D^{\prime}$ to the right of a vertical line between $p_{2 n}$ and $p_{2 n+1}$. There is an embedding

$$
C_{\mathbf{c}^{\prime}}\left(D^{\prime} \backslash D_{2}\right) \times C_{\mathbf{c}_{1}^{\prime}}\left(D_{2}\right) \rightarrow C^{\prime} .
$$

We can assume that $T$ lies in $C_{\mathbf{c}^{\prime}}\left(D^{\prime} \backslash D_{2}\right)$, and

$$
T^{\prime}=T \times T_{n+1},
$$

where $T_{n+1}$ is the obvious $N$-dimensional torus in $C_{\mathbf{c}_{1}^{\prime}}\left(D_{2}\right)$. Then

$$
\iota(\beta)\left(T^{\prime}\right)=\beta(T) \times T_{n+1} .
$$

Equation (2) is now equivalent to

$$
\left\langle Z \times \sigma\left(S_{n+1}\right), \beta(T) \times T_{n+1}\right\rangle=\langle Z, \beta(T)\rangle .
$$

Any point of intersection between $Z \times \sigma\left(S_{n+1}\right)$ and $\beta(T) \times T_{n+1}$ must lie in

$$
C_{\mathbf{c}^{\prime}}\left(D^{\prime} \backslash D_{3}\right) \times C_{\mathbf{c}_{1}^{\prime}}\left(D_{2}\right),
$$

which is the intersection of the two relevant product spaces. Thus it suffices to show

$$
\left\langle\sigma\left(S_{n+1}\right), T_{n+1}\right\rangle=1,
$$

where the intersection pairing is between submanifolds of $C_{\mathbf{c}_{1}^{\prime}}\left(D_{3}\right)$.

All that remains is to compute the intersection pairing of two specific submanifolds of a specific configuration space, depending only on $N$. Figure 9 shows the case $N=1$. The case $N>1$ is similar. There is one point of intersection $\mathbf{y}$ between $\sigma\left(S_{n+1}\right)$ and 


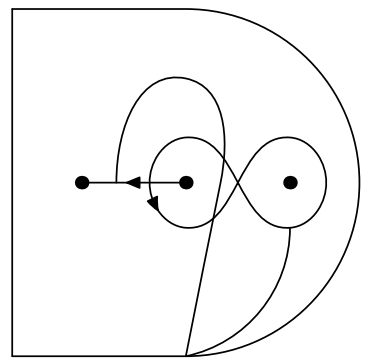

Figure 9: Computing $\left\langle\sigma\left(S_{n+1}\right), T_{n+1}\right\rangle$ when $N=1$

$T_{n+1}$. The sign of this intersection is positive. Each of $\sigma\left(S_{n+1}\right)$ and $T_{n+1}$ comes with a path from a configuration of points on $\partial D_{3}$ to $\mathbf{y}$. These paths are homotopic relative to endpoints. This completes the proof of Equation (3), and hence of the lemma.

\section{The skein relation}

Let $\beta_{+}=\sigma_{2}^{-1} \sigma_{1} \sigma_{2} \beta$ and $\beta_{-}=\sigma_{2}^{-1} \sigma_{1}^{-1} \sigma_{2} \beta$. The aim of this section is to prove the following.

Lemma 10.1 $q^{(N+1) / 2} Q\left(\beta_{-}\right)-q^{-(N+1) / 2} Q\left(\beta_{+}\right)=\left(q^{1 / 2}-q^{-1 / 2}\right) Q(\beta)$.

We have the identities

$$
\begin{aligned}
& \rho\left(\beta_{+}\right)=q^{N / 2} \rho(\beta), \\
& \rho\left(\beta_{-}\right)=q^{-N / 2} \rho(\beta) .
\end{aligned}
$$

Thus it suffices to show

$$
q^{1 / 2}\left\langle S, \beta_{-}(T)\right\rangle-q^{-1 / 2}\left\langle S, \beta_{+}(T)\right\rangle=\left(q^{1 / 2}-q^{-1 / 2}\right)\langle S, \beta(T)\rangle .
$$

Let $Z_{2}$ be as defined in Section 7. By Lemma 7.1, it suffices to show that

$$
q^{1 / 2}\left\langle Z_{2}, \beta_{-}(T)\right\rangle-q^{-1 / 2}\left\langle Z_{2}, \beta_{+}(T)\right\rangle=\left(q^{1 / 2}-q^{-1 / 2}\right)\left\langle Z_{2}, \beta(T)\right\rangle .
$$

By some simple manipulation, this is equivalent to

$$
\left\langle\sigma_{2}^{-1}\left(\sigma_{1}-1\right)\left(\sigma_{1}+q\right) \sigma_{2}\left(Z_{2}\right), \sigma_{2}^{-1} \sigma_{1} \sigma_{2} \beta(T)\right\rangle=0 .
$$

Thus it suffices to prove the identity

$$
\sigma_{2}^{-1}\left(\sigma_{1}-1\right)\left(\sigma_{1}+q\right) \sigma_{2}\left(Z_{2}\right)=0
$$

in $H_{m}^{\ell f}(C, \partial C)$. 
Let $\mathbf{c}_{1}^{\prime}=(1, \ldots, N)$, and let $\mathbf{c}_{2}^{\prime}$ be the $(m-N)$-tuple

$$
\mathbf{c}_{2}^{\prime}=(1, \ldots, N, 1, \ldots, N, \ldots, 1, \ldots, N) .
$$

Thus $\mathbf{c}^{\prime}$ is the concatenation $\mathbf{c}_{1}^{\prime} \mathbf{c}_{2}^{\prime}$.

Let $D_{3}$ be the set of points in $D$ on or to the left of a vertical line between $p_{3}$ and $p_{4}$. There is an obvious embedding

$$
C_{\mathbf{c}_{1}^{\prime}}\left(D_{3}\right) \times C_{\mathbf{c}_{2}^{\prime}}\left(D \backslash D_{3}\right) \rightarrow C .
$$

We can write

$$
Z_{2}=S_{1} \times Z^{\prime}
$$

where $S_{1}$ is the obvious $N$-ball in $C_{\mathbf{c}_{1}^{\prime}}\left(D_{3}\right)$, and $Z^{\prime}$ lies in $C_{\mathbf{c}_{2}^{\prime}}\left(D \backslash D_{3}\right)$.

Now $\sigma_{1}$ and $\sigma_{2}$ both act as the identity on $D \backslash D_{3}$. Thus, to prove Equation (4), it suffices to show that

$$
\sigma_{2}^{-1}\left(\sigma_{1}-1\right)\left(\sigma_{1}+q\right) \sigma_{2}\left(S_{1}\right)=0
$$

in $H_{N}^{\ell f}\left(C_{\mathbf{c}_{1}^{\prime}}\left(D_{3}\right)\right)$.

We now eliminate the conjugation by $\sigma_{2}$ in Equation (5). Let $D_{3}^{\prime}=\sigma_{2} D_{3}$. This is a disk with three puncture points, which have colors $0,0, N+1$, reading from left to right. Let $S_{1}^{\prime}=\sigma_{2} S_{1}$. Then Equation (5) is equivalent to the identity

$$
\left(\sigma_{1}-1\right)\left(\sigma_{1}+q\right)\left(S_{1}^{\prime}\right)=0
$$

in $H_{N}^{\ell f}\left(C_{\mathbf{c}_{1}^{\prime}}\left(D_{3}^{\prime}\right)\right)$.

In the context of $D_{3}^{\prime}$, there are only two nontrivial code sequences up to equivalence. These are $(0,1,2, \ldots, N, 0, N+1)$ and $(0,0,1,2, \ldots, N, N+1)$.

Suppose $Z$ is the barcode corresponding to $(0,1,2, \ldots, N, 0, N+1)$. Then $\sigma_{1}\left(S_{1}^{\prime}\right)$ and $Z$ do not intersect, so

$$
\left\langle\sigma_{1}\left(S_{1}^{\prime}\right), Z\right\rangle^{\prime}=0
$$

Now $S_{1}^{\prime}$ and $Z$ intersect at a single point $\mathbf{y}$. Similarly, $\sigma_{1}^{2}\left(S_{1}^{\prime}\right)$ and $Z$ intersect at a single point, which we can assume is also $\mathbf{y}$. The signs of these intersections are the same. Each of $S_{1}^{\prime}$ and $\sigma_{1}^{2}\left(S_{1}^{\prime}\right)$ comes with a path from $\mathbf{y}$ to $\mathbf{x}$. These paths differ by the direction the points of colors $1, \ldots, N$ pass around the middle puncture point. We obtain the identity

$$
\left\langle S_{1}^{\prime}, Z\right\rangle^{\prime}=q\left\langle\sigma_{1}^{2}\left(S_{1}^{\prime}\right), Z\right\rangle^{\prime} .
$$

A simple computation now gives

$$
\left\langle\left(\sigma_{1}-1\right)\left(\sigma_{1}+q\right)\left(S_{1}^{\prime}\right), Z\right\rangle^{\prime}=0
$$

Algebraic $8 \mathcal{G}$ Geometric Topology, Volume 7 (2007) 
Now suppose $Z$ is the barcode corresponding to $(0,0,1,2, \ldots, N, N+1)$. Then $\sigma_{1}$ acts as the identity on $Z$. It follows that

$$
\left\langle S_{1}^{\prime}, Z\right\rangle^{\prime}=\left\langle\sigma_{1}\left(S_{1}^{\prime}\right), Z\right\rangle^{\prime}=\left\langle\sigma_{1}^{2}\left(S_{1}^{\prime}\right), Z\right\rangle^{\prime} .
$$

A simple computation now gives

$$
\left\langle\left(\sigma_{1}-1\right)\left(\sigma_{1}+q\right)\left(S_{1}^{\prime}\right), Z\right\rangle^{\prime}=0 .
$$

This completes the proof of Equation (6), and hence of the lemma.

\section{Conclusion}

We are now ready to prove the main theorem of this paper. Let $Q(\beta)$ be as defined in Section 4. Let $\widetilde{\beta}$ be the plat closure of $\beta$, as an oriented knot or link. Let $P(\widetilde{\beta})$ be the invariant of $\widetilde{\beta}$ of type $A_{N}$, as defined in the introduction.

Theorem 11.1 $Q(\beta)=P(\tilde{\beta})$.

Proof Birman [3] proved that two braids have isotopic plat closures if and only if they are related by a sequence of moves of certain types. The original theorem applied to unoriented knots, whereas we wish to apply it to oriented knots and links. However the result is essentially the same. The moves are those given in Lemmas 5.1, 8.1, and 9.1. Thus $Q(\beta)$ is an invariant of the oriented knot or link $\widetilde{\beta}$.

Suppose we have three links as shown in the skein relation given at the beginning of this paper. By applying an isotopy, we can present these links as the plat closures of braids $\beta_{+}, \beta_{-}$and $\beta$ as in Section 10. By Lemma 10.1, the invariant $Q$ satisfies the required skein relation.

It remains only to prove that $Q$ is correctly normalized to take the value one for the unknot. Suppose $n=1$ and $\beta$ is the identity braid. By Lemma 6.5,

$$
\langle S, T\rangle=1+q+\cdots+q^{N} .
$$

Thus

$$
Q(\beta)=\frac{1}{[N+1] q^{N / 2}}\left(1+q+\cdots+q^{N}\right)=1
$$

as required.

We now show how to eliminate the factor of $[N+1]$ from the definition of $Q$. Suppose the rightmost strand of $\beta$ makes no crossings with any other strands. Note that any oriented knot or link is the plat closure of some such braid $\beta$. 
Let $m^{\prime}=m-N$. Let $\mathbf{c}_{1}^{\prime}=(1, \ldots, N)$. and let $\mathbf{c}_{2}^{\prime}$ be the $m^{\prime}$-tuple

$$
\mathbf{c}_{2}^{\prime}=(1, \ldots, N, 1, \ldots, N, \ldots, 1, \ldots, N) .
$$

Thus $\mathbf{c}^{\prime}$ is the concatenation $\mathbf{c}_{2}^{\prime} \mathbf{c}_{1}^{\prime}$. Recall that $S$ was defined to be the product of $n$ copies of an $N$-dimensional ball. Let $S^{\prime}$ be the product of all but the rightmost of these $N$-balls, as a subset of $C_{\mathbf{c}_{2}^{\prime}}\left(D_{\mathbf{c}}\right)$. Similarly, let $T^{\prime}$ be the product of all but the rightmost $N$-torus used to define $T$. Let

$$
Q^{\prime}(\beta)=\rho(\beta) q^{-m^{\prime} / 2}\left\langle S^{\prime}, \beta\left(T^{\prime}\right)\right\rangle .
$$

Theorem 11.2 If the rightmost strand of $\beta$ makes no crossings with any other strands then $Q^{\prime}(\beta)=P(\widetilde{\beta})$.

Proof Let $Z_{n}$ be as defined in Section 7. By Lemma 7.1,

$$
\overline{(q-1)}^{N}\langle S, \beta(T)\rangle=\overline{\left(1+q+\cdots+q^{N}\right)}\left\langle Z_{n}, \beta(T)\right\rangle .
$$

Let $D_{1}$ be the once punctured disk consisting of points in $D$ to the right of a vertical line between $p_{2 n-1}$ and $p_{2 n}$. There is an embedding

$$
C_{\mathbf{c}_{2}^{\prime}}\left(D \backslash D_{1}\right) \times C_{\mathbf{c}_{1}^{\prime}}\left(D_{1}\right) \rightarrow C .
$$

Then $Z_{n}=S^{\prime} \times Z$, where $Z$ is the obvious barcode in $C_{\mathbf{c}_{1}^{\prime}}\left(D_{1}\right)$.

Let $D_{2}$ be the twice punctured disk consisting of points in $D$ to the right of a vertical line between $p_{2 n-2}$ and $p_{2 n-1}$. There is an embedding

$$
C_{\mathbf{c}_{2}^{\prime}}\left(D \backslash D_{2}\right) \times C_{\mathbf{c}_{1}^{\prime}}\left(D_{2}\right) \rightarrow C .
$$

Then $T=T^{\prime} \times T_{n}$, where $T_{n}$ is the obvious $N$-torus in $C_{\mathbf{c}_{1}^{\prime}}\left(D_{2}\right)$.

By assumption, $\beta$ acts as the identity on $D_{1}$. Any point of intersection between $Z_{n}$ and $\beta(T)$ must lie in

$$
C_{\mathbf{c}_{2}^{\prime}}\left(D \backslash D_{2}\right) \times C_{\mathbf{c}_{1}^{\prime}}\left(D_{1}\right),
$$

which is the intersection of the two relevant product spaces. Thus

$$
\left\langle Z_{n}, \beta(T)\right\rangle=\left\langle S^{\prime}, \beta\left(T^{\prime}\right)\right\rangle\left\langle Z, T_{n}\right\rangle .
$$

By Lemma 6.4,

$$
\left\langle Z, T_{n}\right\rangle=(1-q)^{N} .
$$

A straightforward calculation now gives $Q^{\prime}(\beta)=Q(\beta)$, as required.

The computational definition of the pairing works over any ring containing an invertible element $q$. Thus $Q^{\prime}(\beta)$ is well defined over any ring containing an invertible element $q^{1 / 2}$. Since it is a polynomial in $q^{ \pm 1 / 2}$, the above theorem applies for any such ring. 


\section{References}

[1] S Bigelow, A homological definition of the Jones polynomial, from: "Invariants of knots and 3-manifolds (Kyoto, 2001)", Geom. Topol. Monogr. 4, Geom. Topol. Publ., Coventry (2002) 29-41 MR2002601

[2] S Bigelow, Homological representations of the Iwahori-Hecke algebra, from: "Proceedings of the Casson Fest", (C Gordon, Y Rieck, editors), Geom. Topol. Monogr. 7, Geom. Topol. Publ., Coventry (2004) 493-507 MR2172492

[3] J S Birman, On the stable equivalence of plat representations of knots and links, Canad. J. Math. 28 (1976) 264-290 MR0402715

[4] R D Budney, On the image of the Lawrence-Krammer representation, J. Knot Theory Ramifications 14 (2005) 773-789 MR2172897

[5] P Freyd, D Yetter, J Hoste, W B R Lickorish, K Millett, A Ocneanu, A new polynomial invariant of knots and links, Bull. Amer. Math. Soc. (N.S.) 12 (1985) 239-246 MR776477

[6] A Hatcher, Algebraic topology, Cambridge University Press, Cambridge (2002) MR1867354

[7] V F R Jones, A polynomial invariant for knots via von Neumann algebras, Bull. Amer. Math. Soc. (N.S.) 12 (1985) 103-111 MR766964

[8] RJ Lawrence, A functorial approach to the one-variable Jones polynomial, J. Differential Geom. 37 (1993) 689-710 MR1217166

[9] R J Lawrence, Braid group representations associated with $\mathfrak{s l}_{m}$, J. Knot Theory Ramifications 5 (1996) 637-660 MR1414092

[10] C Manolescu, Nilpotent slices, Hilbert schemes, and the Jones polynomial, Duke Math. J. 132 (2006) 311-369 MR2219260

[11] P Seidel, I Smith, A link invariant from the symplectic geometry of nilpotent slices, Duke Math. J. 134 (2006) 453-514 MR2254624

Department of Mathematics, University of California at Santa Barbara

California 93106, USA

bigelow@math.ucsb.edu

http://www . math.ucsb.edu/ bigelow/

Received: 23 August 2006 Revised: 14 September 2007 